\title{
Determination of sex using the humerus in a contemporary Sri Lankan population
}

Dissanayake $\mathrm{JK}^{1}$, Kodikara $\mathrm{KAS}^{3}$, Peiris $\mathrm{HRD}^{2}$, Ranasinghe $\mathrm{DJ}^{1}$, Abeywardhana $\mathrm{UNY}^{1} *$, Chandrasiri DACT ${ }^{1}$, Nawarathna $\mathrm{LS}^{4}$, Nanayakkara $\mathrm{CD}^{2}$

${ }^{\prime}$ Department of Anatomy, Faculty of Medicine, University of Peradeniya.

${ }^{2}$ Department of Basic Sciences, Faculty of Dental Sciences, University of Peradeniya.

${ }^{3}$ Department of Forensic Medicine, Faculty of Medicne, University of Peradeniya.

${ }^{4}$ Department of Statistics and Computer Sciences, Faculty of Science, University of

Peradeniya.

*niroabey@yahoo.com

Identification of the biological profile includes determination of sex, age, ethnicity and stature estimation. It is important when unknown skeletal remains are recovered. Metric characteristics of humeri also have received attention in forensic studies for this purpose. The study was conducted in the Department of Anatomy and Department of Forensic Medicine, Faculty of Medicine, and Department of Basic Sciences, Faculty of Dental Sciences, University of Peradeniya. A sample of 136 dry humeri was studied. Six measurements were taken including the weight, maximum length, circumference at the proximal $1 / 3$, middle $1 / 3$, distal $1 / 3$ and the length of the shaft using standard techniques. Descriptive statistics were obtained separately for each sex. Two sample t-test was used to assess whether there is a significant difference between males and females. Univariate and stepwise discriminant function analyses were performed to assess the sex classification potential of humeral measurements. The data sets were normally distributed. All 6 dimensions were statistically greater in males $(p<0.05)$. Weight measurement gives the highest accuracy of sex classification with an accuracy of $83.3 \%$. Circumference at the middle $1 / 3$ and distal $1 / 3$ also gives more than $80 \%$ accuracy of sex determination. The best multivariate equation for the sample was the combination of weight and circumference at the proximal $1 / 3$ with a cross validated accuracy of $82 \%$ and $84.7 \%$ original group accuracy. Sex determination is important to narrow down the possible identities of the unknown skeletal remains. The humerus has a good sex predicting potential for the Sri Lankan contemporary population. 